ZIBELINE INTERNATATIONAL

ISSN: 2521-0874 (Print)

ISSN: 2521-0505 (online)

CODEN : AIMCCO

\title{
A DESIGN OF WSN BASED LOCKING SYSTEM
}

\author{
Haixiang Tao, Shang Zhang, Cifa Chen \\ College of Computer and Information Technology China Three Gorges University Yi Chang, China. \\ *Corresponding Author Email: wetoo@163.com
}

This is an open access article distributed under the Creative Commons Attribution License, which permits unrestricted use, distribution, and reproduction in any medium, provided the original work is properly cited.

\section{ARTICLE DETAILS}

Article History:

Received 10 December 2017

Accepted 08 January 2018

Available online 19 February 2018

\begin{abstract}
This paper studied and designed a smart lock based on STM32F4 and CC2530 wireless module and proposed the overall design scheme of the system. By utilize the wireless transmission characteristics of ZigBee module, we can make the STM32F4 as the main control chip, and CC2530 as the wireless transmission module to realize the control of smart door lock. The smart lock consists of a smart lock device and a CC2530 wireless transmission module. The structure of the intelligent lock device mainly includes the module of door lock control chip, monitoring module, lock tongue drive module and power supply circuit. The intelligent lock device uses the STM32F4 microcontroller as the door lock control chip, sends an unlock instruction to the CC2530 module A by using the USART serial port, and then sends the received instruction to the CC2530 module B connected with the smart lock by the CC2530 module A. Control the opening and closing of the smart lock device.
\end{abstract}

\section{KEYWORDS}

Smart lock, wireless module, wireless transmission, microcontroller, intelligent lock

\section{INTRODUCTION}

With the development of modern science and technology, the "lock" is not only a tool to lock the door, but also an intelligent tool that embodies the wisdom. The lock has been developed from the traditional mechanical lock to the intelligent pattern, however, the smart lock still has the following problems in the development process:

1.) The passwords are easily leaked, the unlocking is slow, and it will take longer time if we input the error password, in addition, it will be less secure in the process of entering passwords with people who are next to you.

2.) Contact IC card -smart lock card which application is less and less, only used in the occasions which are related with the magnetic stripe card, is easy to damage and lost.

3.) The cost of IC card of the Non-contact IC card smart lock is so high that it is not suitable for large-scale application. And there are certain security risks as the information is easy to be copied.

Our system adopts CC2530 wireless module to transmit unlocking and locking signals, overcoming the problem of inputting the password and making the IC card, improving the safety and practicality of the door lock. and each module has low cost and low power consumption, so it is suitable for large-scale application.

\section{SYSTEM DESIGN}

\subsection{Principle of system}

to the door. The unlocking part contains the door, lock and the CC2530 module B. Served as the slave communication end, the CC2530 module can transmit the information of the control part to the door lock and utilize the control circuit inside the lock body to change the opening and closing of the lock.

\subsection{The system structures}

The CC2530 intelligent door lock is mainly composed of control unit and locking end, in which the control unit contains STM32F4 one-chip computer, CC2530 module A, and the locking end includes: body of locking body and CC2530 module B and so on. As shown in Figure 1, the control unit sends the AT signal which inputs by the user through the main communication module CC2530 A, then the slave communication module CC2530 B receives the AT signal sent by A.

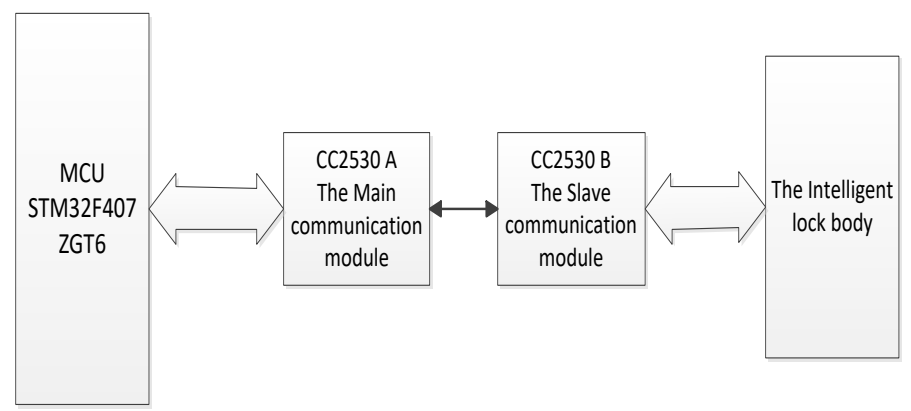

Figure 1: Intelligent lock structure diagram

When the users need to open the door, only send the unlock signal to the control chip by the serial port debugging assistant, STM32F4 will send the signal to the main communication module CC2530A through UART1, then, through $\mathrm{A}$ to transmit the unlock signal to the slave communication module B, After received the signal sent by A, B would unlock the lock, Through the control circuit inside the lock to realize unlock action.

\section{THE HARDWARE CIRCUIT}

\subsection{STM32F4 Microcontroller}

STM32F4 with FPU and DSP instruction sets adopts the Cortex M4 kernel, and possesses up to 192KB of SRAM with Camera Interface (DCMI), cryptographic Processor (CRYP)、high-Speed USB OTG, true Random Number Generator, OTP memory, faster A / D conversion speed, lower ADC / DAC working voltage, 32-bit timer, 6 serial ports, real-time clock with calendar (RTC), IO multiplexing function greatly enhanced, $4 \mathrm{~K}$ bytes of battery backup SRAM As well as faster USART and SPI communication speed. Its maximum operating frequency can reach to $168 \mathrm{Mhz}$.

\subsection{CC2530 module}

In this paper, we designed the CC2530 1A wireless communication 
module by hand using discrete elements which would be easy to fabricate. As shown in figure 2 .
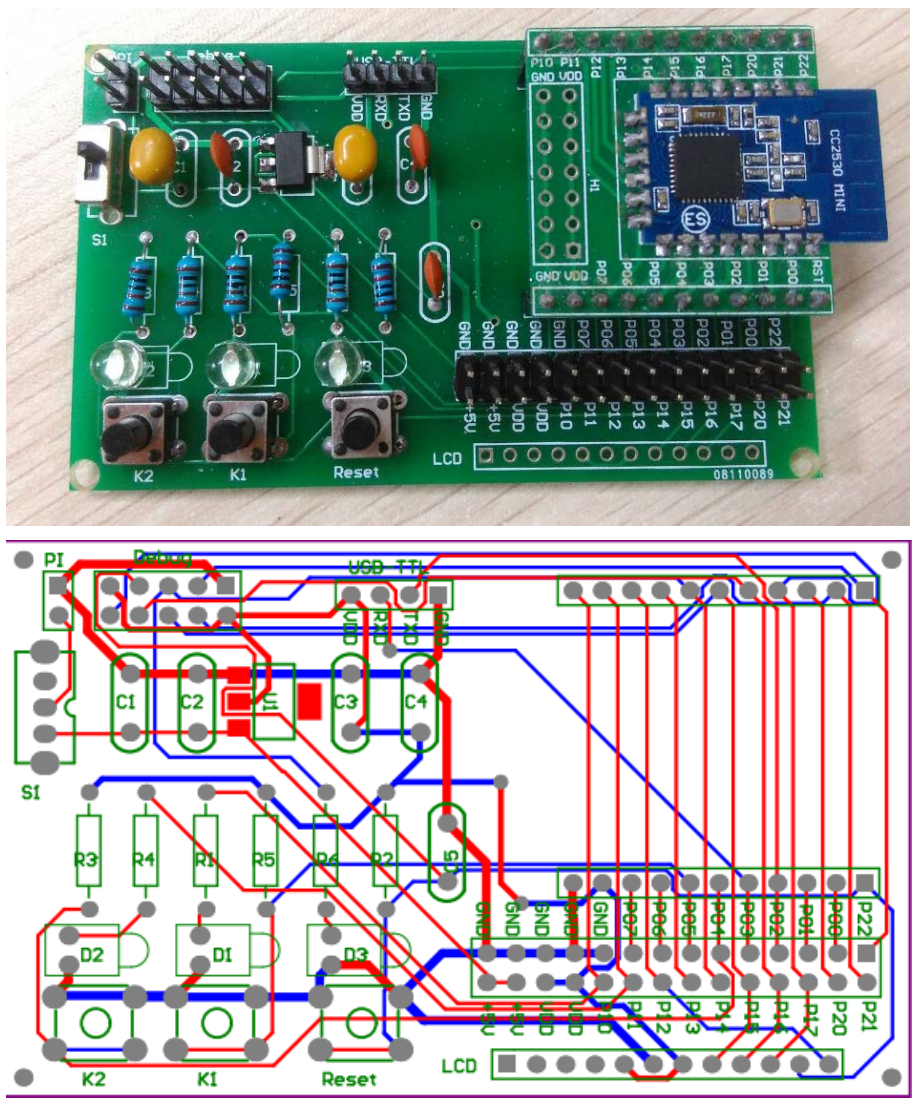

Figure 2: CC2530 1A wireless communication module

The core of the module consists of MINI CC2530 chip, power supply circuit, the download debugging interface, the serial port to the USB conversion circuit, and the reset circuit. Among them, the MINI CC2530 ZigBee module has a small size $2.7 \mathrm{~dB}$ patch ceramic antenna, which in addition can send and receive signals at the same time, enabling longdistance wireless communications with low power consumption.

Developed product locks. As shown in Figure 3.

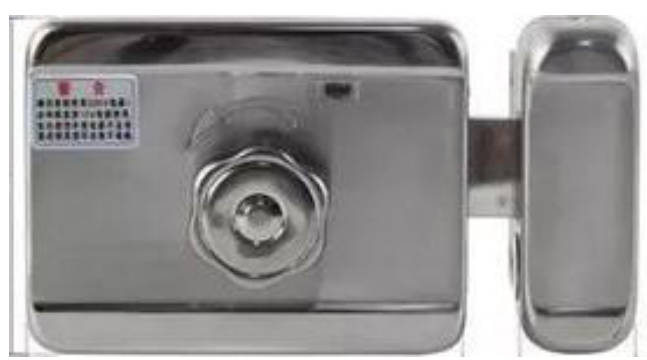

Figure 3: The overall diagram of smart locks

The metal body which can resist damage and rust is safe and reliable. Power Control Module. Shown in Figure 4

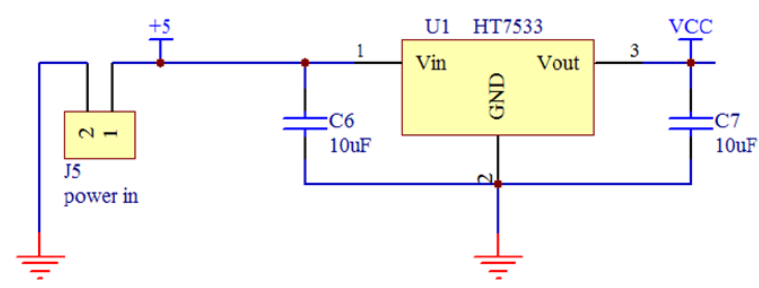

Figure 4: Power Supply Module

The power supply module with $220 \mathrm{~V}$ input will adjust the voltage required by each module by the transformer. The standard voltage of CC2530 input is $3.3 \mathrm{~V}$. In the smart lock terminal, the external power source is selected from J5 access to select the micro-power supply management chip, the HT7533 will adapt the power supply voltage Mach with that of CC2530.

\section{SOFTWARE DESIGN}

\subsection{Software architecture}

This software system includes three main functional modules: user terminal module, background management module and device management module. The specific structure diagram is shown in Figure 5

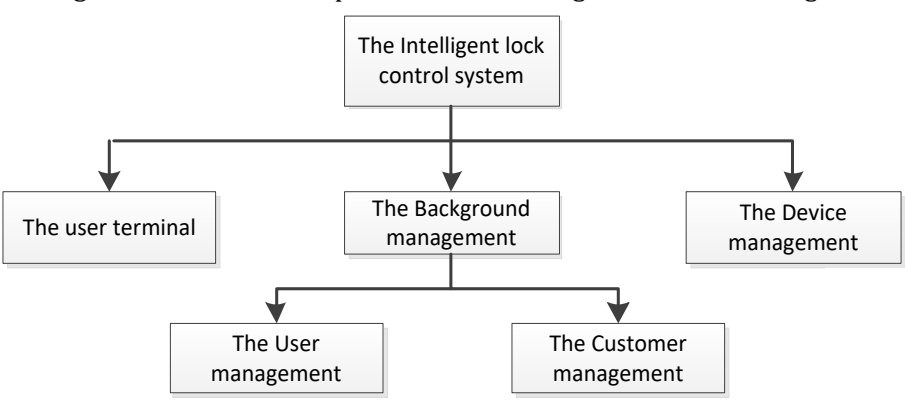

Figure 5: Software overall functional structure diagram

The User terminal module: users establish communication with the phone through the WIFI module controlled by STM32F4. It can issue the turn-on or turn-off signals to STM32F4 through WIFI module on the mobile APP When you need to open the lock or shut the lock. The AT instruction is sent to CC2530 A module which dispose the received signal and forwarded it to reach the B module. Finally, CC2530 B module control the opening and closing of smart lock.

Background management module: which establish the back-end database, store and manage the user's and customer's information such as authorization and record the time that the user accesses the system, so it is convenient for user to see about the record.

Device module: It is designed to detect the operation of intelligent lock When the lock fails in function, it can automatically send information to the backstage for maintenance person and maintenance of the Device.

\subsection{User module design}

User module: Used for user to log in account on the mobile APP. The specific flow chart shown in Figure 6.

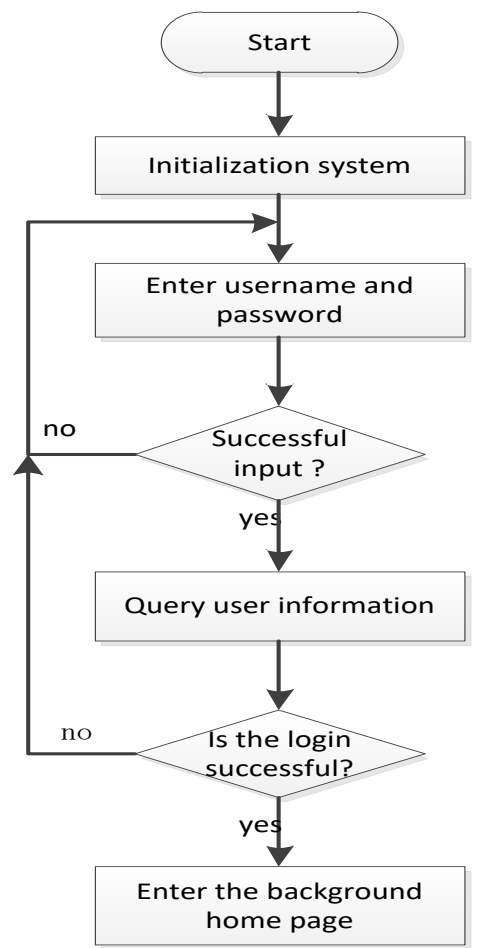

Figure 6: User module running flow chart

When the users $\log$ in the intelligent lock control system, initialize the system firstly, enter the user name and password interface with a delay of a few microseconds. After the users enter the account number and password, the system automatically detects whether it is successful. Then it will enter the next step: a query of user's information if the input is successful: the system will automatically match the username and password entered in the database with the back-end 
database. If the matching succeeds, the user will enter the background control interface, otherwise, the user is warned to re-enter the username and password.

\section{CONCLUSIONS}

In this paper, we first designed a wireless control lock based on STM32F4 and CC2530 module. This device with a small size, low power consumption and low price not only can solve some problems that the intelligent lock posed but also a small intelligent device suitable for civilian use that meets the smart locks of the average household and small warehouse. What is more, it can effectively reduce the risk of theft of goods in household and warehouse, meaningful to the real life.

\section{ACKNOWLEDGEMENTS}

This paper is supported by the CERNET through Project "NGII20161210".

\section{REFERENCE}

[1] Liu, C.M., Chen, L.S. 2009. Applications of RFID technology for improving production efficiency in an integrated-circuit packaging house [J]. International Journal of Production Research Electronics, 47 (8), 2203-2216.

[2] Aqeel, R., Abbasi, A.Z. 2008. Building A Smart University using RFID Technology [J]. International Conference on Computer Science and Software Engineering, 641-644.

[3] Kim, H., Chung, J.M., Kim, C.H. 2009. Zig Bee Secured communication protocol for internetworking Zig Bee cluster networks [J]. Computer Communications, 32 (13-14), 1531-1540.
[4] Huircán, J.I., Muñoz, C., Young, H., Dossow, L.V., Bustos, J., Vivallo, G., Toneatti, M. 2010. Zig Bee-based wireless sensor network localization for cattle monitoring in grazing fields [J]. Computers and Electronics in Agriculture, 74 (2), 258-264.

[5] Bo, J., Lianbo, M., Jiawang, X. 2010. Research of the Collection System of Logistics Management Based on RFID \& Zig Bee Technology[J]. International Conference on Computer Application and System Modelling, 526-529.

[6] Shang, Z., Tingyan, X., Liufeng, T. 2013. OSGIS \& FOSS in LBS design, Proceedings of the 2013 International Conference on Software Engineering and Computer Science, ISBN: 978-90786-77-82-6, 09, P.261.

[7] Zhang, S., Xing, T., Tao, L. 2014. FOSS-based WSN indoor localization platform design, International Conference on GIS and Resource Management. ISBN : 978-1-60595-147-8, 01, P401-408

[8] Zhang, S., Xing, T. 2013. Open WSN indoor localization platform design, Instrumentation and Measurement, Sensor Network and Automation (IMSNA), 2nd International Symposium on, Digital Object, EI检 索, ISBN:978-1-4799-2716-6, 12, P845.

[9] Tingyan, X., Zhang, S., Zhang, F. 2014. RSSI Enhanced Microkernel-Based LBS Design. Journal of Geographic Information System, 4, 109. ISSN : 2151-1950.

[10] Zhang, S., Xing, T. 2014. RSSI Enhanced indoor LBS platform Design. Computer Modelling \& New Technologies, EI检索, ISSN:1407-5806, 05.

[11] Xing, T., Zhang, S., Zhang, F. 2014. Microkernel and Middle-Ware Based GIS Platform Design. Journal of Positioning, ISSN: 2150-850X, 05, P53. 